\title{
EXISTENCE RESULT FOR A NEW CLASS OF KIRCHHOFF ELLIPTIC SYSTEM WITH VARIABLE PARAMETERS
}

\author{
MOHAMED HAIOUR, SALAH BOULAARAS, RAFIK GUEFAIFIA, \\ AND FARES KAMACHE
}

Received 08 June, 2020

\begin{abstract}
The paper studies the existence result for a new class of Kirchhoff elliptic system with variable parameters in the right hand side. Sub-super solutions method are used for proving the main result. Our study is a natural improvement result of our previous one in (Boulaaras et al. in Math. Methods Appl. Sci. 41:5203-5210, 2018).
\end{abstract}

2010 Mathematics Subject Classification: 35J60; 35B30; 35B40

Keywords: Kirchhoff elliptic systems, existence, positive solutions, sub-supersolution, variable parameters

\section{INTRODUCTION}

Consider the following system

$$
\left\{\begin{array}{l}
-A\left(\int_{\Omega}|\nabla u|^{2} d x\right) \triangle u=\lambda \alpha(x) f(u, v) \text { in } \Omega \\
-B\left(\int_{\Omega}|\nabla v|^{2} d x\right) \triangle v=\lambda \beta(x) g(u, v) \text { in } \Omega \\
u=v=0 \text { on } \partial \Omega
\end{array}\right.
$$

where $\Omega \subset \mathbb{R}^{N}(N \geq 3)$ is a bounded smooth domain with $C^{2}$ boundary $\partial \Omega$, and $A$, $B: \mathbb{R}^{+} \rightarrow \mathbb{R}^{+}$are continuous functions with further conditions to be given later, $\lambda$ is a positive parameter, and $\alpha, \beta \in C(\bar{\Omega})$.

The second author would like to thank his Professors/Scientists Pr. Mohamed Haiour and Pr. Azzedine Benchettah for the important masters and $\mathrm{PhD}$ courses content in pure and applied mathematics which he received them during his studies. Moreover, he thanks them for the additional help they provided him during office hours in their office about the few concepts/difficulties He had encountered and appreciated their talent and dedication for their postgraduate students currently and previously. 
This nonlocal problem originates from the stationary version of Kirchhoff's work [10] in 1883

$$
\rho \frac{\partial^{2} u}{\partial t^{2}}-\left(\frac{P_{0}}{h}+\frac{E}{2 L} \int_{0}^{L}\left|\frac{\partial u}{\partial x}\right|^{2} d x\right) \frac{\partial^{2} u}{\partial x^{2}}=0,
$$

where Kirchhoff extended the classical d'Alembert's wave equation by considering the effect of the changes in the length of the string during vibrations. The parameters in (1.2) have the following meanings: $L$ is the length of the string, $h$ is the area of the cross-section, $E$ is the Young modulus of the material, $\rho$ is the mass density, and $P_{0}$ is the initial tension.

Recently, the problems associated to Laplacian operator and Kirchhoff elliptic equations have been heavily studied, we refer to $[1,3-5,8,9,11-13]$.

In [2], Alves and Correa proved the validity of Sub-super solutions method for problems of Kirchhoff class involving a single equation and a boundary condition

$$
\left\{\begin{aligned}
-M\left(\|u\|^{2}\right) \Delta u & =f(x, u) \text { in } \Omega, \\
u & =0 \quad \text { on } \partial \Omega,
\end{aligned}\right.
$$

with $f \in C(\bar{\Omega} \times \mathbb{R})$.

By using a comparison principle that requires $M$ to be non-negative and nonincreasing in $[0,+\infty)$, with $H(t):=M\left(t^{2}\right) t$ increasing and $H(\mathbb{R})=\mathbb{R}$, they managed to prove the existence of positive solutions assuming $f$ increasing in the variable $u$ for each $x \in \Omega$ fixed.

For systems involving similar class of equations, this result can not be used directly, i.e. the existence of a subsolution and a supersolution does not guarantee the existence of the solution. Therefore, a further construction is needed. As in [6], where we studied the system

$$
\left\{\begin{array}{l}
-A\left(\int_{\Omega}|\nabla u|^{2} d x\right) \triangle u=\lambda_{1} f(v)+\mu_{1} g(u) \text { in } \Omega, \\
-B\left(\int_{\Omega}|\nabla v|^{2} d x\right) \Delta v=\lambda_{2} h(u)+\mu_{2}(x) l(v) \text { in } \Omega, \\
u=v=0 \text { on } \partial \Omega .
\end{array}\right.
$$

Using a weak positive supersolution as first term of a constructed iterative sequence $\left(u_{n}, v_{n}\right)$ in $H_{0}^{1}(\Omega) \times H_{0}^{1}(\Omega)$, and a comparison principle introduced in [2], the authors established the convergence of this sequence to a positive weak solution of the considered problem.

In this paper, we generalize the previous work in [6] by considering variable parameters $\alpha, \beta, \gamma$ and $\eta$ in the right hand side of (1.1). We also give a better subsolution providing easier computations. 


\section{EXISTENCE RESULT}

Definition 1. $(u, v) \in\left(H_{0}^{1}(\Omega) \times H_{0}^{1}(\Omega)\right)$, is called a weak solution of (1.1) if it satisfies

$$
\begin{aligned}
& A\left(\int_{\Omega}|\nabla u|^{2} d x\right) \int_{\Omega} \nabla u \nabla \phi d x=\lambda \int_{\Omega} \alpha(x) f(u, v) \phi d x \text { in } \Omega, \\
& B\left(\int_{\Omega}|\nabla v|^{2} d x\right) \int_{\Omega} \nabla v \nabla \psi d x=\lambda \int_{\Omega} \beta(x) g(u, v) \psi d x \text { in } \Omega,
\end{aligned}
$$

for all $(\phi, \psi) \in\left(H_{0}^{1}(\Omega) \times H_{0}^{1}(\Omega)\right)$.

Definition 2. Let $(\underline{u}, \underline{v}),(\bar{u}, \bar{v})$ be a pair of nonnegative functions in $\left(H_{0}^{1}(\Omega) \times H_{0}^{1}(\Omega)\right)$, they are called positive weak subsolution and positive weak supersolution (respectively) of (1.1) if they satisfy the following

$$
\begin{aligned}
& A\left(\int_{\Omega}|\nabla \underline{u}|^{2} d x\right) \int_{\Omega} \nabla \underline{u} \nabla \phi d x \leq \lambda \int_{\Omega} \alpha(x) f(\underline{u}, \underline{v}) \phi d x, \\
& B\left(\int_{\Omega}|\nabla \underline{v}|^{2} d x\right) \int_{\Omega} \nabla \underline{v} \nabla \psi d x \leq \lambda \int_{\Omega} \beta(x) g(\underline{u}, \underline{v}) \psi d x,
\end{aligned}
$$

and

$$
\begin{aligned}
& A\left(\int_{\Omega}|\nabla \bar{u}|^{2} d x\right) \int_{\Omega} \nabla \bar{u} \nabla \phi d x \geq \lambda \int_{\Omega} \alpha(x) f(\bar{u}, \bar{v}) \phi d x, \\
& B\left(\int_{\Omega}|\nabla \bar{v}|^{2} d x\right) \int_{\Omega} \nabla \bar{v} \nabla \psi d x \geq \lambda \int_{\Omega} \beta(x) g(\bar{u}, \bar{v}) \psi d x,
\end{aligned}
$$

for all $(\phi, \psi) \in\left(H_{0}^{1}(\Omega) \times H_{0}^{1}(\Omega)\right)$, with $\phi \geq 0$ and $\psi \geq 0$, and $(\underline{u}, \underline{v}),(\bar{u}, \bar{v})=(0,0)$ on $\partial \Omega$.

Lemma 1 (Comparison principle [2]). Let $M: \mathbb{R}^{+} \rightarrow \mathbb{R}^{+}$be a continuous nonincreasing function such that

$$
M(s)>m_{0}>0, \text { for all } s \geq s_{0},
$$

and $H(t)=t M\left(t^{2}\right)$ increasing on $\mathbb{R}^{+}$. 
If $u_{1}, u_{2}$ are two non-negative functions verifying

$$
\left\{\begin{array}{l}
-M\left(\int_{\Omega}\left|\nabla u_{1}\right|^{2} d x\right) \triangle u_{1} \geq-M\left(\int_{\Omega}\left|\nabla u_{2}\right|^{2} d x\right) \triangle u_{2} \text { in } \Omega \\
u_{1}=u_{2}=0 \text { on } \partial \Omega
\end{array}\right.
$$

then $u_{1} \geq u_{2}$ a.e. in $\Omega$.

Before stating and proving our main result, here are the conditions we need.

(H1) $A, B: \mathbb{R}^{+} \rightarrow \mathbb{R}^{+}$are two continuous and increasing functions that satisfy the monotonicity conditions of Lemma 1 so that we can use the Comparison principle, and assume further that there exists $a_{i}, b_{i}>0, i=1,2$,

$$
a_{1} \leq A(t) \leq a_{2}, \quad b_{1} \leq B(t) \leq b_{2} \text { for all } t \in \mathbb{R}^{+} .
$$

(H2) $\alpha, \beta \in C(\bar{\Omega})$ and

$$
\alpha(x) \geq \alpha_{0}>0, \beta(x) \geq \beta_{0}>0
$$

for all $x \in \Omega$.

(H3) $f, g$ are continuous on $\left[0,+\infty\left[, C^{1}\right.\right.$ on $(0,+\infty)$, and increasing functions of infinite growth

$$
\lim _{s, t \rightarrow+\infty} f(s, t)=+\infty, \lim _{s, t \rightarrow+\infty} g(s, t)=+\infty .
$$

(H4) For all $K>0$

$$
\lim _{t \rightarrow+\infty} \frac{f(t, K(g(t, t)))}{t}=0
$$

$$
\lim _{t \rightarrow+\infty} \frac{g(t, t)}{t}=0
$$

Theorem 1. For large values of $\lambda \alpha_{0}$ and $\lambda \beta_{0}$, system (1.1) admits a large positive weak solution if conditions $(H 1)-(H 5)$ are satisfied.

Proof of Theorem 1. Consider $\sigma$ the first eigenvalue of $-\triangle$ with Dirichlet boundary conditions and $\phi_{1}$ the corresponding positive eigenfunction with $\left\|\phi_{1}\right\|=1$ and $\phi_{1} \in C^{\infty}(\bar{\Omega})($ see $[7])$.

Let $S=\sup _{x \in \Omega}\left\{\sigma \phi_{1}^{2}-\left|\nabla \phi_{1}\right|^{2}\right\}$, then from growth conditions (H3)

$$
f(t, t) \geq S, g(t, t) \geq S, \quad \text { for } t \text { large enough. }
$$

For each $\alpha_{0}$ large, let us define

$$
\underline{u}=\left(\frac{\lambda \alpha_{0}}{2 a_{2}}\right) \phi_{1}^{2}
$$


and

$$
\underline{v}=\left(\frac{\lambda \beta_{0}}{2 b_{2}}\right) \phi_{1}^{2}
$$

where $a_{2}, b_{2}$ are given by condition $(H 1)$. Let us show that $(\underline{u}, \underline{v})$ is a subsolution of problem (1.1) for $\lambda \alpha_{0}$ large enough. Indeed, let $\phi \in H_{0}^{1}(\Omega)$ with $\phi \geq 0$ in $\Omega$. By $(H 1)-(H 3)$, we get

$$
\begin{aligned}
& A\left(\int_{\Omega}|\nabla \underline{u}|^{2} d x\right) \int_{\Omega} \nabla \underline{u} . \nabla \phi d x=A\left(\int_{\Omega}|\nabla \underline{u}|^{2} d x\right)\left(\frac{\lambda \alpha_{0}}{a_{2}}\right) \int_{\Omega} \phi_{1} \nabla \phi_{1} \cdot \nabla \phi d x \\
& =\left(\frac{\lambda \alpha_{0}}{a_{2}}\right) A\left(\int_{\Omega}|\nabla \underline{u}|^{2} d x\right) \times\left\{\int_{\Omega} \nabla \phi_{1} \nabla\left(\phi_{1} . \phi\right) d x-\int_{\Omega}\left|\nabla \phi_{1}\right|^{2} \phi d x\right\} \\
& =\left(\frac{\lambda \alpha_{0}}{a_{2}}\right) A\left(\int_{\Omega}|\nabla \underline{u}|^{2} d x\right) \int_{\Omega}\left(\sigma \phi_{1}^{2}-\left|\nabla \phi_{1}\right|^{2}\right) \phi d x \\
& \leq \lambda \alpha_{0} \int_{\Omega} S \phi d x \leq \lambda \int_{\Omega} \alpha(x) f(\underline{u}, \underline{v}) \phi d x
\end{aligned}
$$

for $\lambda \alpha_{0}>0$ large enough, and all $\phi \in H_{0}^{1}(\Omega)$ with $\phi \geq 0$ in $\Omega$.

Similarly,

$$
B\left(\int_{\Omega}|\nabla \underline{v}|^{2} d x\right) \int_{\Omega} \nabla \underline{v} \nabla \psi d x \leq \lambda \int_{\Omega} \beta(x) g(\underline{u}, \underline{v}) \psi d x \text { in } \Omega
$$

for $\lambda \beta_{0}>0$ large enough and all $\psi \in H_{0}^{1}(\Omega)$ with $\psi \geq 0$ in $\Omega$.

Also notice that $\underline{u}>0$ and $\underline{v}>0$ in $\Omega, \underline{u} \rightarrow+\infty$ and $\underline{v} \rightarrow+\infty$ as $\lambda \alpha_{0} \rightarrow+\infty$, $\lambda \beta_{0} \rightarrow+\infty$.

For the supersolution part, consider $e$ the solution of the following problem

$$
\left\{\begin{array}{c}
-\triangle e=1 \text { in } \Omega \\
e=0 \text { on } \partial \Omega .
\end{array}\right.
$$

We give the supersolution of problem (1.1) by

$$
\bar{u}=\frac{C}{\mu}\left(\lambda\|\alpha\|_{\infty}\right) e, \quad \bar{v}=\left(\frac{\lambda\|\beta\|_{\infty}}{b_{2}}\right) g(C \lambda, C \lambda) e,
$$

where $\mu=\|e\|_{\infty}, C>0$ is a large positive real number to be given later.

Indeed, for all $\phi \in H_{0}^{1}(\Omega)$ with $\phi \geq 0$ in $\Omega$, we get from (2.3) and the condition (H1)

$$
A\left(\int_{\Omega}|\nabla \bar{u}|^{2} d x\right) \int_{\Omega} \nabla \bar{u} . \nabla \phi d x=A\left(\int_{\Omega}|\nabla \bar{u}|^{2} d x\right) \frac{C}{\mu}\left(\lambda\|\alpha\|_{\infty}\right) \int_{\Omega} \nabla e . \nabla \phi d x
$$




$$
=A\left(\int_{\Omega}|\nabla \bar{u}|^{2} d x\right) \frac{C \lambda}{\mu}\left(\|\alpha\|_{\infty}\right) \int_{\Omega} \phi d x \geq \frac{a_{1} C \lambda}{\mu}\left(\|\alpha\|_{\infty}\right) \int_{\Omega} \phi d x .
$$

By $(H 4)$ and $(H 5)$, we can choose $C$ large enough so that

$$
\frac{a_{1} C \lambda}{\mu} \int_{\Omega} \phi d x \geq \lambda \int_{\Omega} f\left(C \lambda,\left(\frac{\lambda\|\beta\|_{\infty}}{b_{2}}\right) g(C \lambda, C \lambda) \mu\right) \phi d x .
$$

Therefore,

$$
\begin{aligned}
A\left(\int_{\Omega}|\nabla \bar{u}|^{2} d x\right) \int_{\Omega} \nabla \bar{u} . \nabla \phi d x & \geq \lambda\|\alpha\|_{\infty} \int_{\Omega} f\left(C \lambda,\left(\frac{\lambda\|\beta\|_{\infty}}{b_{2}}\right) g(C \lambda, C \lambda) \mu\right) \phi d x \\
& \geq \lambda\|\alpha\|_{\infty} \int_{\Omega} f\left(\frac{C}{\mu} \lambda e,\left(\frac{\lambda\|\beta\|_{\infty}}{b_{2}}\right) g(C \lambda, C \lambda) e\right) \phi d x \\
& \geq \lambda \int_{\Omega} \alpha(x) f(\bar{u}, \bar{v}) \phi d x
\end{aligned}
$$

Also,

$$
\begin{aligned}
B\left(\int_{\Omega}|\nabla \bar{v}|^{2} d x\right) \int_{\Omega} \nabla \bar{v} \nabla \psi d x & =\frac{\lambda\|\beta\|_{\infty}}{b_{2}} g(C \lambda, C \lambda) B\left(\int_{\Omega}|\nabla \bar{v}|^{2} d x\right) \int_{\Omega} \nabla e \nabla \psi d x \\
& \geq \lambda\|\beta\|_{\infty} g(C \lambda, C \lambda) \int_{\Omega} \psi d x
\end{aligned}
$$

Using (H4) and (H5) again for $C$ large enough we get

$$
\frac{1}{\frac{\lambda\|\beta\|_{\infty}}{b_{2}} \mu} \geq \frac{g(C \lambda, C \lambda)}{C \lambda}
$$

Hence

$$
\begin{aligned}
g(C \lambda, C \lambda) \int_{\Omega} \psi d x & \geq \int_{\Omega} g\left(C \lambda, g(C \lambda, C \lambda) \frac{\left(\lambda\|\beta\|_{\infty}\right)}{b_{2}} \mu\right) \psi d x \\
& \geq \int_{\Omega} g\left(\lambda C\left(\frac{e}{\mu}\right), g(C \lambda, C \lambda) \frac{\left(\lambda\|\beta\|_{\infty}\right)}{b_{2}} e\right) \psi d x \\
& =\int_{\Omega} g(\bar{u}, \bar{v}) \psi d x
\end{aligned}
$$


Combining (2.5) and (2.6), we obtain

$$
B\left(\int_{\Omega}|\nabla \bar{v}|^{2} d x\right) \int_{\Omega} \nabla \bar{v} \nabla \psi d x \geq \lambda \int_{\Omega} \beta(x) g(\bar{u}, \bar{v}) \psi d x .
$$

By (2.4) and (2.7) we conclude that $(\bar{u}, \bar{v})$ is a supersolution of problem (1.1). Furthermore, $\underline{u} \leq \bar{u}$ and $\underline{v} \leq \bar{v}$ for $C$ choosen large enough.

Now, we use a similar argument to [6] in order to obtain a weak solution of our problem. Consider the following sequence $\left\{\left(u_{n}, v_{n}\right)\right\} \subset\left(H_{0}^{1}(\Omega) \times H_{0}^{1}(\Omega)\right)$ where: $u_{0}:=\bar{u}, v_{0}=\bar{v}$ and $\left(u_{n}, v_{n}\right)$ is the unique solution of

$$
\left\{\begin{array}{l}
-A\left(\int_{\Omega}\left|\nabla u_{n}\right|^{2} d x\right) \triangle u_{n}=\lambda \alpha(x) f\left(u_{n-1}, v_{n-1}\right) \text { in } \Omega, \\
-B\left(\int_{\Omega}\left|\nabla v_{n}\right|^{2} d x\right) \triangle v_{n}=\lambda \beta(x) g\left(u_{n-1}, v_{n-1}\right) \text { in } \Omega, \\
u_{n}=v_{n}=0 \text { on } \partial \Omega .
\end{array}\right.
$$

Since $A$ and $B$ satisfy $(H 1)$ and $\alpha(x) f\left(u_{n-1}, v_{n-1}\right), \beta(x) g\left(u_{n-1}, v_{n-1}\right) \in L^{2}(\Omega)$ (in $x$ ), we deduce from a result in [2] that system (2.8) has a unique solution $\left(u_{n}, v_{n}\right) \in$ $\left(H_{0}^{1}(\Omega) \times H_{0}^{1}(\Omega)\right)$.

Using (2.8) and the fact that $\left(u_{0}, v_{0}\right)$ is a supersolution of (1.1), we get

$$
\left\{\begin{array}{c}
-A\left(\int_{\Omega}\left|\nabla u_{0}\right|^{2} d x\right) \triangle u_{0} \geq \lambda \alpha(x) f\left(u_{0}, v_{0}\right)=-A\left(\int_{\Omega}\left|\nabla u_{1}\right|^{2} d x\right) \triangle u_{1}, \\
-B\left(\int_{\Omega}\left|\nabla v_{0}\right|^{2} d x\right) \triangle v_{0} \geq \lambda \beta(x) g\left(u_{0}, v_{0}\right)=-B\left(\int_{\Omega}\left|\nabla v_{1}\right| d x\right) \triangle v_{1}
\end{array}\right.
$$

then by Lemma $1, u_{0} \geq u_{1}$ and $v_{0} \geq v_{1}$. Also, since $u_{0} \geq \underline{u}, v_{0} \geq \underline{v}$ and the monotonicity of $f, g, h$, and $l$ one has

$$
\begin{gathered}
-A\left(\int_{\Omega}\left|\nabla u_{1}\right|^{2} d x\right) \triangle u_{1}=\lambda \alpha(x) f\left(u_{0}, v_{0}\right) \geq \lambda \alpha(x) f(\underline{u}, \underline{v}) \geq-A\left(\int_{\Omega}|\nabla \underline{u}|^{2} d x\right) \triangle \underline{u}, \\
-B\left(\int_{\Omega}\left|\nabla v_{1}\right|^{2} d x\right) \triangle v_{1}=\lambda \beta(x) g\left(u_{0}, v_{0}\right) \geq \lambda \beta(x) g(\underline{u}, \underline{v}) \geq-B\left(\int_{\Omega}|\nabla \underline{v}|^{2} d x\right) \triangle \underline{v}
\end{gathered}
$$

according to Lemma 1 again, we obtain $u_{1} \geq \underline{u}, v_{1} \geq \underline{v}$. Repeating the same argument for $u_{2}, v_{2}$, observe that

$$
-A\left(\int_{\Omega}\left|\nabla u_{1}\right|^{2} d x\right) \triangle u_{1}=\lambda \alpha(x) f\left(u_{0}, v_{0}\right) \geq \lambda \alpha(x) f\left(u_{1}, v_{1}\right)=-A\left(\int_{\Omega}\left|\nabla u_{2}\right|^{2} d x\right) \triangle u_{2},
$$




$$
-B\left(\int_{\Omega}\left|\nabla v_{1}\right| d x\right) \triangle v_{1}=\lambda \beta(x) g\left(u_{0}, v_{0}\right) \geq \lambda \alpha(x) g\left(u_{1}, v_{1}\right)=-B\left(\int_{\Omega}\left|\nabla v_{2}\right|^{2} d x\right) \triangle v_{2},
$$

then $u_{1} \geq u_{2}, v_{1} \geq v_{2}$. Similarly, we get $u_{2} \geq \underline{u}$ and $v_{2} \geq \underline{v}$ from

$$
\begin{gathered}
-A\left(\int_{\Omega}\left|\nabla u_{2}\right|^{2} d x\right) \triangle u_{2}=\lambda \alpha(x) f\left(u_{1}, v_{1}\right) \geq \lambda \alpha(x) f(\underline{u}, \underline{v}) \geq-A\left(\int_{\Omega}|\nabla \underline{u}|^{2} d x\right) \triangle \underline{u}, \\
-B\left(\int_{\Omega}\left|\nabla v_{2}\right|^{2} d x\right) \triangle v_{2}=\lambda \beta(x) g\left(u_{1}, v_{1}\right) \geq \lambda \beta(x) g(\underline{u}, \underline{v}) \geq-B\left(\int_{\Omega}|\nabla \underline{v}|^{2} d x\right) \triangle \underline{v} .
\end{gathered}
$$

By repeating these implementations we construct a bounded decreasing sequence $\left\{\left(u_{n}, v_{n}\right)\right\} \subset\left(H_{0}^{1}(\Omega) \times H_{0}^{1}(\Omega)\right)$ verifying

$$
\begin{aligned}
& \bar{u}=u_{0} \geq u_{1} \geq u_{2} \geq \ldots \geq u_{n} \geq \ldots \geq \underline{u}>0, \\
& \bar{v}=v_{0} \geq v_{1} \geq v_{2} \geq \ldots \geq v_{n} \geq \ldots \geq \underline{v}>0 .
\end{aligned}
$$

By continuity of functions $f, g, h$, and $l$ and the definition of the sequences $\left(u_{n}\right)$ and $\left(v_{n}\right)$, there exist positive constants $C_{i}>0, i=1, \ldots, 4$ such that

$$
\left|f\left(u_{n-1}, v_{n-1}\right)\right| \leq C_{1}, \quad\left|g\left(u_{n-1}, v_{n-1}\right)\right| \leq C_{2} \text { for all } n .
$$

From (2.11), multiplying the first equation of (2.8) by $u_{n}$, integrating, using Holder inequality and Sobolev embedding we check that

$$
\begin{aligned}
& a_{1} \int_{\Omega}\left|\nabla u_{n}\right|^{2} d x \leq A\left(\int_{\Omega}\left|\nabla u_{n}\right|^{2} d x\right) \int_{\Omega}\left|\nabla u_{n}\right|^{2} d x=\lambda \int_{\Omega} \alpha(x) f\left(u_{n-1}, v_{n-1}\right) u_{n} d x \\
& \leq \lambda\|\alpha\|_{\infty} \int_{\Omega}\left|f\left(u_{n-1}, v_{n-1}\right)\right|\left|u_{n}\right| d x \leq C_{1} \int_{\Omega}\left|u_{n}\right| d x \leq C_{3}\left\|u_{n}\right\|_{H_{0}^{1}(\Omega)}
\end{aligned}
$$

or

$$
\left\|u_{n}\right\|_{H_{0}^{1}(\Omega)} \leq C_{3}, \forall n
$$

where $C_{3}>0$ is a constant independent of $n$. Similarly, there exist $C_{4}>0$ independent of $n$ such that

$$
\left\|v_{n}\right\|_{H_{0}^{1}(\Omega)} \leq C_{4}, \quad \forall n
$$

From (2.12) and (2.13), we deduce that $\left\{\left(u_{n}, v_{n}\right)\right\}$ admits a weakly converging subsequence in $H_{0}^{1}\left(\Omega, \mathbb{R}^{2}\right)$ to a limit $(u, v)$ satisfying $u \geq \underline{u}>0$ and $v \geq \underline{v}>0$. Being monotone and also using a standard regularity argument, $\left\{\left(u_{n}, v_{n}\right)\right\}$ converges itself to $(u, v)$. Now, letting $n \rightarrow+\infty$ in (2.13), we conclude that $(u, v)$ is a positive weak solution of system (1.1). 


\section{ACKNOWLEDGMENT}

The authors are grateful to the anonymous referees for the careful reading and their important observations/suggestions for the sake of improving this paper. In memory of Mr. Mahmoud ben Mouha Boulaaras (1910-1999).

\section{REFERENCES}

[1] M. Alizadeh and M. Alimohammady, "Regularity and entropy solutions of some elliptic equations." Miskolc Mathematical Notes., vol. 19, no. 2, pp. 715-729, 2018, doi: 10.18514/MMN.2018.2545.

[2] C. Alves and F. J. S. A. Correa, "On existence of solutions for a class of problem involving a nonlinear operator." Communications on Applied Nonlinear Analysis., vol. 8, no. 2, pp. 43-56, 2001.

[3] N. Azouz and A. Bensedik, "Existence result for an elliptic equation of Kirchhoff -type with changing sign data." Funkcial. Ekvac., vol. 55, pp. 55-66, 2012.

[4] Y. Bouizem, S. Boulaaras, and B. Djebbar, "Some existence results for an elliptic equation of Kirchhoff-type with changing sign data and a logarithmic nonlinearity." Mathematical methods in the applied sciences., vol. 42, no. 7, pp. 2465-2474, 2019, doi: 10.1002/mma.5523.

[5] Y. Bouizem, S. Boulaaras, and B. Djebbar, "Existence of positive solutions for a class of Kirchhof elliptic systems with right hand side defined as a multiplication of two separate functions." Kragujevac journal of mathematics., vol. 45, no. 4, pp. 587-596, 2021.

[6] S. Boulaaras and R. Guefaifia, "Existence of positive weak solutions for a class of Kirrchoff elliptic systems with multiple parameters." Math. Methods Appl., vol. 41, pp. 5203-5210, 2018, doi: 10.1002/mma.5071.

[7] L. Evans and F. J. S. A. Correa, "Partial differential equations.” American Mathematical Society, Providence., 2010.

[8] S. Gala, Q. Liu, and M. A. Ragusa, "A new regularity criterion for the nematic liquid crystal fows." Applicable Analysis., vol. 91, no. 9, pp. 1741-1747, 2012, doi: 10.1080/00036811.2011.581233.

[9] S. Gala and M. Ragusa, "Logarithmically improved regularity criterion for the Boussinesq equations in Besov spaces with negative indices." Applicable Analysis., vol. 95, no. 6, pp. 1271-1279, 2016, doi: 10.1080/00036811.2015.1061122.

[10] G. Kirchhoff, Mechanik and Teubner, Leipzig, 1883.

[11] S. Polidoro and M. A. Ragusa, "Harnack inequality for hypoelliptic ultraparabolic equa- tions with a singular lower order term." Revista Matematica Iberoamericana., vol. 24, no. 3, pp. 1011-1046, 2008, doi: 10.4171/RMI/565.

[12] B. Ricceri, "On an elliptic Kirchhoff -type problem depending on two parameters." J. Global Optim., vol. 46, pp. 543-549, 2010.

[13] J. Sun and C. L. Tang, "Existence and multiplicity of solutions for Kirchhoff type equations." Nonlinear Anal., vol. 74, pp. 1212-1222, 2011, doi: 10.1016/j.na.2010.09.061.

Authors' addresses

Mohamed Haiour

LANOS Laboratory, Faculty of Sciences, Badji Mokhtar-Annaba University, 23000 Annaba, Algeria

E-mail address: haiourmeyahoo.com 
Salah Boulaaras

Department of Mathematics, College of Sciences and Arts in Al-Rass, Qassim University, Buraidah, 51452 Buraidah, Saudi Arabia

Laboratory of Fundamental and Applied Mathematics of Oran (LMFAO), University of Oran 1, Oran, 31000 Oran, Algeria

E-mail address: s.boularas@qu.edu.sa

Rafik Guefaifia

Laboratory of Mathematics, Informatics and systemes (LAMIS), Larbi Tebessi University, Algeria, 12000 Tebessa, Algeria

E-mail address: rafikik982@gmail.com

Fares Kamache

Department of Mathematics, Faculty of Exact Sciences, University Tebessa, Tebessa, Algeria, 12000 Tebessa, Algeria

E-mail address: fares.kamache@univ-tebessa.dz 